\title{
Is the nasal route a viable option for relieving acute pain in pediatric emergency medicine? A literature review
}

\author{
Nabil Abdulrahman Aleysae \\ Department of Pediatrics, King Faisal Specialist Hospital and Research Centre, Jeddah, Saudi Arabia
}

\begin{abstract}
In recent years, the nasal route has increasingly been viewed as an alternative option for the delivery of analgesia, especially when the traditional ways are complicated or time-intensive. However, little is known about the value of this intervention in acute pain management in pediatric emergency medicine.

This evidence-based analysis review aims to assess the current evidence regarding the use, safety, and effectiveness of intranasal analgesics in acutely painful conditions encountered in Pediatric Emergency Departments (PEDs).

A systemic electronic searching of Cochrane library, PubMed, and EMBASE databases from the beginning of each database until October 2018 was conducted using a maximally sensitive searching strategy. Only randomized controlled trials (RCTs) or quasirandomized controlled trials that evaluated the use of intranasal analgesia for acute pain in children in the Emergency Department and published between January 1990 and October 2018 were included. The methodological quality of the trials was assessed using the Grading of Recommendations Assessment, Development, and Evaluation criteria. Risks of bias within each
\end{abstract}

Correspondence: Nabil Abdulrahman Aleysae, Department of Pediatrics, King Faisal Specialist Hospital and Research Centre, Jeddah, Saudi Arabia.

Tel. 0126677777 , ext. 63516

E-mail: nal-esaei@kfshrc.edu.sa

Acknowledgments: the author acknowledges the support of his tutor Dr. Thomas Beattie, University of Edinburgh, for organizing and supervising the course of the project and the article, as well as his encouragement in carrying out this college work.

Key words: Intranasal; Analgesia; Acute pain; Pediatric emergency medicine.

Conflict of interest: the author declares no potential conflict of interest.

Funding: none.

Received for publication: 31 May 2019.

Revision received: 6 July 2019.

Accepted for publication: 17 September 2019.

This work is licensed under a Creative Commons Attribution 4.0 License (by-nc 4.0).

(C) Copyright: the Author(s), 2019

Licensee PAGEPress, Italy

Emergency Care Journal 2019; 15:8320

doi:10.4081/ecj.2019.8320 included study were evaluated according to the Cochrane Risk of Bias Tool for RCTs. This review was reported following the Preferred Reporting Items for Systematic Reviews and MetaAnalyses statement.

Seven RCTs and one quasi-randomized study met the inclusion criteria. Five studies compared an intranasal analgesic and an alternative intervention, two compared intranasal fentanyl against ketamine, and one compared two different concentrations of intranasal fentanyl. All included trials reported reductions in pain scores, especially within the first 10 to 30 minutes post-intervention; however, pain reduction was maintained to 60 minutes in only one study. No evidence of significant adverse events was associated with the administration of any intranasal analgesic in any of the included studies.

This review identified eight articles that discussed the intranasal analgesia as a possible route of analgesia in the PED. While no paper was entirely perfect, the findings support the idea that intranasal analgesia may be an effective analgesic for the treatment of children (3-18 years) with acute moderate to severe pain, and its administration appears to cause minimal adverse effects.

\section{Introduction}

Acute pain is one of the significant symptoms and pervasive source of suffering for children presenting to the Pediatric Emergency Departments (PEDs). ${ }^{1,2}$ The prevalence of acute pain in children who present to the PED had been reported as presenting complain in $41 \%$ of children who transported by emergency ambulance to four tertiary referral hospitals in a recent study in Ireland. ${ }^{3}$ Of 334 children older than four years who studied in another pilot study, $48 \%$ had severe pain. ${ }^{1}$

Acute pain in Emergency Department (ED) can be a result of several medical conditions (trauma, injuries, burn, or painful diseases) as well as simple venipuncture and surgical interventions. ${ }^{4}$ Murphy et al. found that 2071 out of 2635 (78\%) of acute pain episodes resulted from traumatic injuries, while non-traumatic conditions reported in $20 \%$ of cases. ${ }^{3}$

It has been recognized that sever unrelieved pain has long-term consequences on a child's behavior such as increased anxiety, decreased pain tolerance, and fear of future medical visits..$^{5-8}$ The literature also indicates that children may suffer posttraumatic stress disorder symptoms after painful and stressful procedures in the PED. ${ }^{9}$ Furthermore, the lack of appropriate and effective analgesia in the ED had associated with higher admission rates, more extended hospitalization, and higher cost to patients and organizations..$^{10}$ Therefore, adequate pain control had recommended in several clinical practice guidelines in pediatric emergency medicine..$^{11,12}$

Although several advances have been made in improving pediatric pain management, ${ }^{13}$ timely management of acute pain in children continues to be suboptimal in both prehospital and PED. ${ }^{14-16}$ 
The difficulty of assessing pain in young children, the unfamiliarity of medical staff with new products and techniques, fear of adverse medication effects, staffing limitations, and time constraints ${ }^{17,18}$ are the significant barriers to adequate pain management in PEDs. Additionally, some healthcare providers still have been believing that neonates and infants feel less pain than adults, making their pain management ineffectively. ${ }^{19}$

Optimal pain management in children requires both non-pharmacological interventions and adequate administration of pain medications. Different distraction techniques, oral sucrose administration to the neonates, cutaneous stimulation, elevation and immobilization of a fractured limb, and applying protective dressings to burns have shown to have a beneficial effect during different procedures in the PED, especially in younger age groups. ${ }^{20-22}$ The administration of systemic analgesia is warranted whenever non-pharmacological approaches are insufficient, or not achieved the needed pain relief lonely. The main aim of systemic analgesic administration is the establishment of useful pain relieving at the first attempt through using an appropriate drug, dose, and route without causing more pain. ${ }^{23}$ Nonsteroidal anti-inflammatory drugs and opioids remain the most commonly used drugs for controlling moderate to severe pain in PEDs. ${ }^{24}$

Multiple options for delivering systemic analgesia have practiced in pediatric medicine, including oral, rectal, topical, subcutaneous, mucosal, parenteral, and inhalation. Despite having many advantages, these traditional routes are not always appropriate or feasible, particularly in PED and prehospital settings. Therefore, the availability of an alternative way, thereby providing analgesia rapidly and safely is an attractive route. The nasal cavity is an easily accessible vascular bed that has many features making it a lovely route for analgesic administration. Six arterial branches serve the nasal cavity, which makes it a much-vascularized surface. ${ }^{25}$ Its venous return drains to the internal jugular vein which in turn flows into the right heart chambers, ${ }^{26,27}$ enables the intranasal absorbed drug to avoid the gastrointestinal degradation and hepatic first-pass metabolism, resulting in a rapid onset of action similar to those being by intravenous (IV) administration and better than subcutaneous (SC), intramuscular (IM), and rectal one. ${ }^{28,29}$ Moreover, the nasal cavity is lined by a mucous membrane that covered by numerous microvilli and its subepithelial cells are bound by a fenestrated epithelium, features converting it to a large and suitable area for fast and reliable drug absorption which minimize the lag time associated with oral drug delivery. ${ }^{26,30}$ Furthermore, unlike parenteral drug therapy, nasal drug administration is a simple, painless, non-invasive, self-used, and convenient method. ${ }^{31}$

This review aims to assess the current evidence regarding the use, safety, and effectiveness of intranasal (IN) analgesia in the treatment of the children who presented with acute moderate to severe pain to the PEDs.

\section{Methods of research}

\section{Design}

This literature review was conducted to identify and evaluate all randomized controlled trials (RCTs) and quasi-randomized trials (QRTs) that assessed the analgesic efficacy of one or more IN analgesic(s) in children who presented with acute moderate to severe pain. The retrieved studies were not sufficiently homogeneous to design systemic review and meta-analysis, so, only qualitative analysis was conducted. This review was reported following the Preferred Reporting Items for Systematic Reviews and MetaAnalyses statement. ${ }^{32}$

\section{Inclusion and exclusion criteria}

All RCTs and QRTs that compared one (or more) IN analgesic agent(s) against a placebo, other IN analgesic, or against alternative analgesic intervention for relieving of acute pain of children in PED or prehospital setting, were included. Only articles published in English, conducted in humans, and published between January 1990 and October 2018 were selected. Conference abstracts, case reports, narrative reviews, editorials, comments, dissertations, animal studies, unpublished or unavailable in English, studies not contained sufficient details regarding the primary outcomes; and those evaluated the efficacy of IN analgesia in a setting outside the PED were excluded. The inclusion and exclusion criteria are summarized in Table 1.

\section{Search strategy}

A systemic electronic searching of the Cochrane library, PubMed, and EMBASE databases from the beginning of each database until October 2018 was conducted using a maximally sensitive strategy to identify all relevant literature. Search strategies for each database were shown in Appendix 1. The search strategy was adapted for each database as required, using Boolean operators and wildcards to account for variations across databases. Several keywords including intranasal analgesics, nasal, intranasal, analgesia, acute pain, pain, procedural, young child, chil-

Table 1. Inclusion and exclusion criteria.

\begin{tabular}{|c|c|c|c|}
\hline Item & Inclusion criteria & Exclusion criteria & Comments \\
\hline Time span & 01 Jan 1990 - 31 October 2018 & Before Jan 1990; after October 2018 & - \\
\hline Age limits & Aged 1-18 years & $<1$ year; over 18 years & $\begin{array}{l}\text { One study has a mixed population } \\
\text { (children and adults) was excluded }\end{array}$ \\
\hline Language & English & Other languages & $\begin{array}{l}\text { When the full report had not available } \\
\text { in English it had been excluded }\end{array}$ \\
\hline Type of study & RCTs and quasi-randomized trials & $\begin{array}{l}\text { Case reports, retrospective, cohort, } \\
\text { case-control, and narrative review }\end{array}$ & $\begin{array}{l}\text { Only studies conducted in humans } \\
\text { were included }\end{array}$ \\
\hline Type of publication & Full report & $\begin{array}{l}\text { Conference abstracts, editorials, } \\
\text { comments, dissertations, } \\
\text { and unpublished studies }\end{array}$ & - \\
\hline Setting & $\begin{array}{l}\text { Trials conducted in EDs and/or } \\
\text { prehospital settings }\end{array}$ & $\begin{array}{l}\text { Studies conducted in setting } \\
\text { outside the EDs }\end{array}$ & $\begin{array}{l}\text { Studies conducted in mixed ED } \\
\text { (pediatric and adults) were included }\end{array}$ \\
\hline
\end{tabular}

RCTs, randomized controlled trials; EDs, emergency departments. 
dren, pediatric, emergency department, and pre-hospital setting were used independently as well as in various combinations. Moreover, nasal or intranasal term matching with specific medication including fentanyl, sufentanil, alfentanil, remifentanil, ketamine, dexmedetomidine, diamorphine, butorphanol as well as buprenorphine were used too. After that, hand-searching of the reference lists of relevant articles was done to identify other potential references and grey literature.

\section{Population}

Children less than 18 years old who presented with an acute pain severity caused by either bone fractures, burns, wounds, emergency medical procedures, or medical illness; who received at least one dose of intranasal analgesia in the PED. This review excluded patients who used IN analgesia for the treatment of postoperative pain in operation rooms, the pain of dental procedures in dental clinics, or as a pretreatment before endoscopies. Patients who received IN analgesics in a setting outside the ED or for indications other than analgesia were also excluded.

\section{Outcome measures}

The primary outcome is to determine if the intranasal delivery of analgesia is as effective as other routes of drug delivery in providing analgesia in the PED through comparison to the reduction in pain as measured by a recognized pain score. The minimum clinically significant differences (MCSD) in pain score, which selected by the authors and considered as the cutoff value for establishing the therapeutic importance of the results, were deemed to be significant when they had achieved.

The secondary outcomes were to determine the rate of failure with IN drug delivery as determined by the rate of rescue medication and to compare rates of adverse events with IN drug delivery.

\section{Data management, collection, and analysis}

Once collected, the obtained articles were exported to Mendeley Desktop bibliographic software for storage. After removal of duplicates, each title and abstract of the remaining studies were assessed for relevance. The full copies of all relevant studies were screened and evaluated for selection according to the inclusion criteria. Principles of the Grading of Recommendation, Assessment, Development, and Evaluation system were used to assess the methodological quality of each trial. ${ }^{33}$ Risks of bias within each included study were evaluated according to the Cochrane Risk of Bias Tool for RCTs. ${ }^{34}$ The following criteria were taken into consideration: random sequence generation; allocation concealment; blinding of personnel, participants and outcome assessment; incomplete outcome data; selective reporting; and other bias. The relevant data were extracted from the selected studies using a pre-specified data extraction form (Appendix 2) which recorded many specified items like the methodological character of the study, participant's characteristics, inclusion and exclusion criteria, main features of intervention and comparison agents, and relevant outcomes. The descriptive data were tabulated within tables, and after that, the consistent findings brought together as a narrative review.

\section{Results}

\section{Results of the search}

The primary search of electronic databases and other sources yielded a total of 363 publications. After the removal of duplicates and screening of the titles and abstracts of all remaining studies, 27 full papers were retrieved for possible inclusion. When the full texts had been examined, 19 articles were excluded, and only eight randomized trials met the inclusion criteria. Figure 1 summarizes the study selection process. This review was performed based on those eight articles. ${ }^{35-42}$

Included studies were published between 1999 and 2018, and were conducted in Australia $(n=4)$, United State $(n=2)$, and the United Kingdom $(n=2)$. All included studies contained two comparison arms. Three IN analgesics were evaluated in these clinical trials: fentanyl (INF), diamorphine (IND), and ketamine (INK). Borland (2007), ${ }^{35}$ Kendal, ${ }^{37}$ Younge, ${ }^{40}$ Fenster, ${ }^{41}$ and Wilson ${ }^{42}$ compared IN analgesics $v s$ alternative interventions, while Graudins ${ }^{38}$ and Reynolds ${ }^{39}$ compared INF against INK, and Borland (2011) $)^{36}$ compared two different concentrations of INF. Table 2 showed the main methodology characteristics of included studies.

A typically included study asked verbal children to score their pain intensity immediately before the administration of the study drug and then at multiple time points after the intervention. Every subject was shown age-appropriate pain scale and asked to rank his pain by pointing to the face that he feels is most consistent with his current pain level, and verbalizing the number that corresponds to their pain on the numeric rating scale. Pain intensity at each subsequent follow-up point was compared with the baseline reading to measure the amount of pain reduction.

Six studies described the reduction in pain intensity over time as the primary outcome measure while remaining ${ }^{39,40}$ as a secondary outcome. With the exception of Fenster et al.,$^{41}$ all the investigators ask the patients, their parents, and/or attending physician/nurse to measured pain intensity at 0-time (baseline) and compared it to several follow-up measurements (e.g., at 5, 10, 15, 20, 30, or 60 minutes) post IN drug administration. Instead, Fenster et al. assigned an Observational Scale of Behavioral Distress-revised (OSBD-R) score to each of the following predetermined phases of abscess incision and drainage: i) pre-analgesic

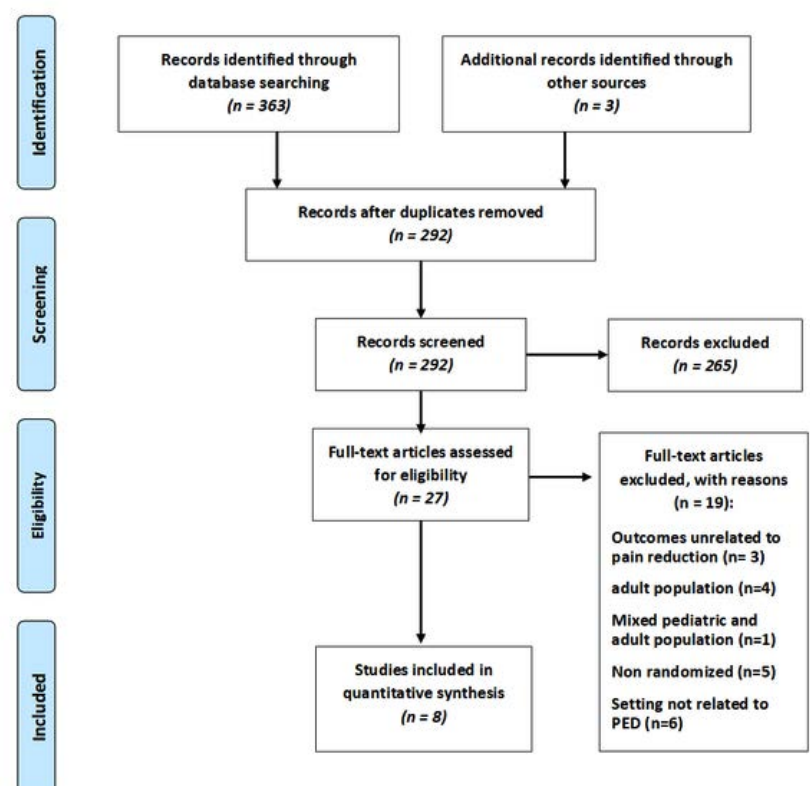

Figure 1. Study selection process. Flow chart of retrieved, excluded and analyzed trials. PED, Pediatric Emergency Department. 


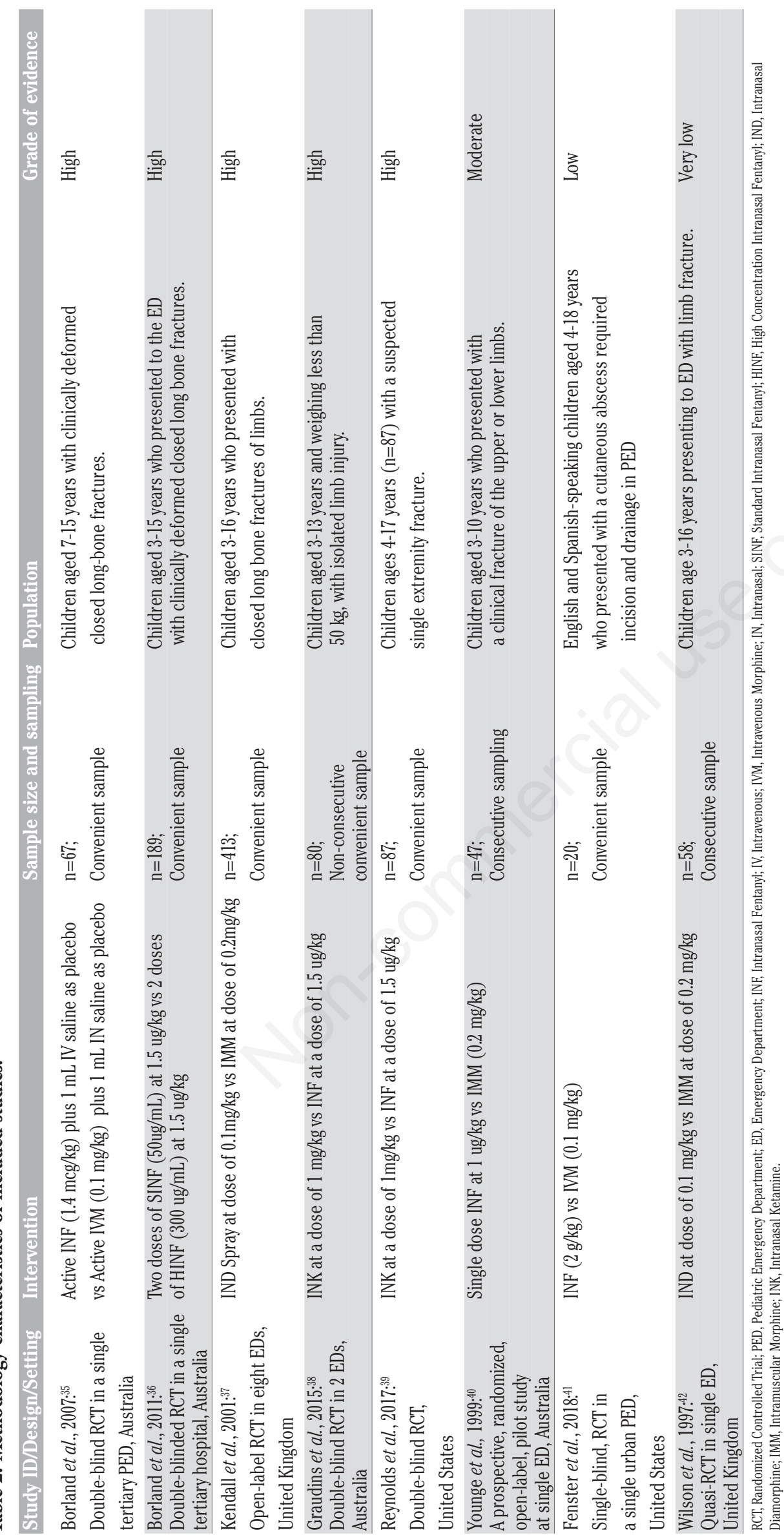


administration; ii) 10 minutes after analgesic administration but pre-procedural; iii) lidocaine infiltration; iv) skin incision; v) abscess drainage; and vi) 10 minutes after procedure completion. All trials assessed pain intensity by single or combined validated scales. Seven studies reported results as the difference between the median/mean pain scores of both groups at each time point, while one study compared and reported the cumulative percentages of patients' pain scores over time according to Wong-Baker Faces (WBF) pain scale. ${ }^{37}$ The participant's tolerance to the drug administration was reported in four studies, $, 35,37,40,41$ and the occurrence of adverse events and assessment of the failure rate of IN analgesia after administration was documented in all included trials. This outcome was reported as a number or percentage of the patient who needs rescue analgesia during the emergency room stay.

\section{Patients' characteristics}

The patient population in the included trials was heterogeneous. Sample sizes were varied widely, ranging from 20 to 413 patients. Each of the eight selected studies evaluated IN analgesia in pediatric patients (aged between 3 to 18 years) who had presented with moderate to severe acute pain to the EDs. All trials investigated pain in suspected limb fractures except Fenster who studied pain of cutaneous abscess incision and drainage. All the trials reported comparable baseline characteristics and presenting pain between control and intervention arms. Exclusion criteria found to be similar for all included studies (e.g., no consent, head injury or trauma impairing judgment, known allergy to opiates, blocked/traumatized nose, parenteral or IN opioid analgesic use before arrival, participants requiring immediate IV access, and inability to perform pain scoring).

\section{Risk of bias in included studies}

The included trials had various methodological methods (Table 2 ). Four trials conducted as double-blind, two as open-labeled, one as single labeled, and one as a quasi-RCT. Judgments about each bias item for every study were summarized in Figures 2 and 3.

\section{Effects of interventions}

A summary of the main findings of the included trials is presented in Table 3 .

\section{Reduction in pain score}

All included trials reported significant reductions in pain scores especially within the first 10 to 30 minutes post-intervention, however, pain reduction was maintained to 60 minutes in only one study for both intervention agents, IN fentanyl (INF) and IN ketamine (INK). ${ }^{38}$

Borland et al. (2011) $)^{36}$ found an equivalent effect in reducing pain when two doses of standard INF ( $50 \mathrm{ugs} / \mathrm{mL}$ ) were compared with 2 doses of highly concentrated (300 ugs $/ \mathrm{mL})$ at dose of 1.5 $\mathrm{ug} / \mathrm{kg}$, with the trend to increased oral additional agents in the more diluted solution. Each intervention demonstrated a statistically and clinically significant decrease in pain scores (median reduction for both groups $40 \mathrm{~mm}, \mathrm{P}=0.000$ ) over the study time (at 10 , 20- and 30-minutes) post initial dose.

Some studies comparing INF to parenteral morphine (IV or IM) and reported that INF was an effective alternative to the use of morphine in the management of pain in children who had limb fractures or procedure pain. Both Younge $e^{40}$ and Fenster ${ }^{41}$ is favoring INF against morphine. Younge reported a significant reduction in pain scores at $10 \mathrm{~min}$ after INF administration. The median
WBF pain score was 1 in the INF group vs 2 in IM morphine $(\mathrm{P}=0.014)$, while no significant difference observed in other time points. Despite this analgesic effect of INF, which lasted for 30 min period, the analgesia was not perfect, as only $11 \%$ of children were pain-free at $10 \mathrm{~min}$ and $22 \%$ at $30 \mathrm{~min}$. Likewise, Fenster reported that INF was superior to IV morphine for the procedural analgesia as a whole as well as for the lidocaine infiltration and abscess drainage phases while it was non-inferior during abscess incision and 10 minutes post procedure phases. The total mean OSBD-R was 5.48 in INF vs 19.9 for IVM (mean difference: -13.4, 1-tailed $97.5 \%$ CI: -24.24 to -2.67 ). In contrast, in his high-quality, randomized, and double-blind trial, Borland (2007) ${ }^{35}$ found no statistically significant differences in Visual Analogue Scale (VAS)

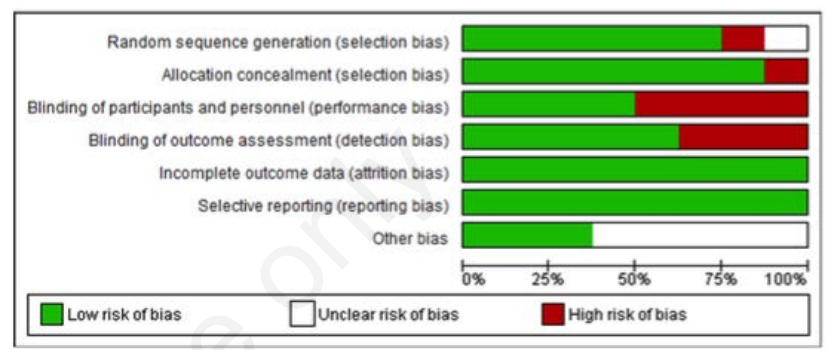

Figure 2. Risk of bias: review authors' judgments about each risk of bias item presented as percentages across all included studies.

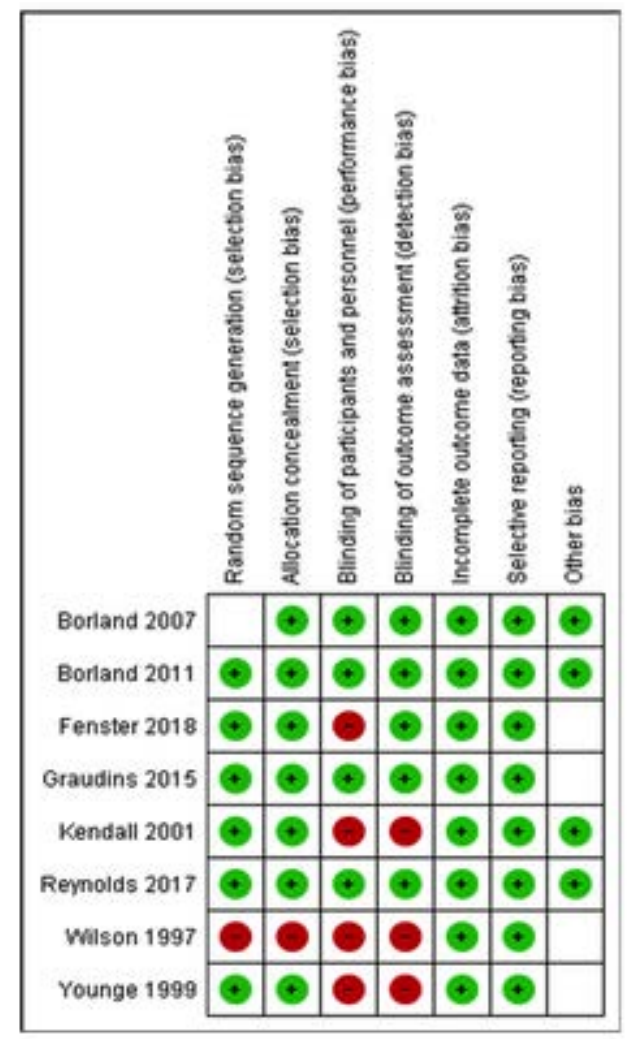

Figure 3. Risk of bias summary: review authors' judgments about each risk of bias item for each included study. 


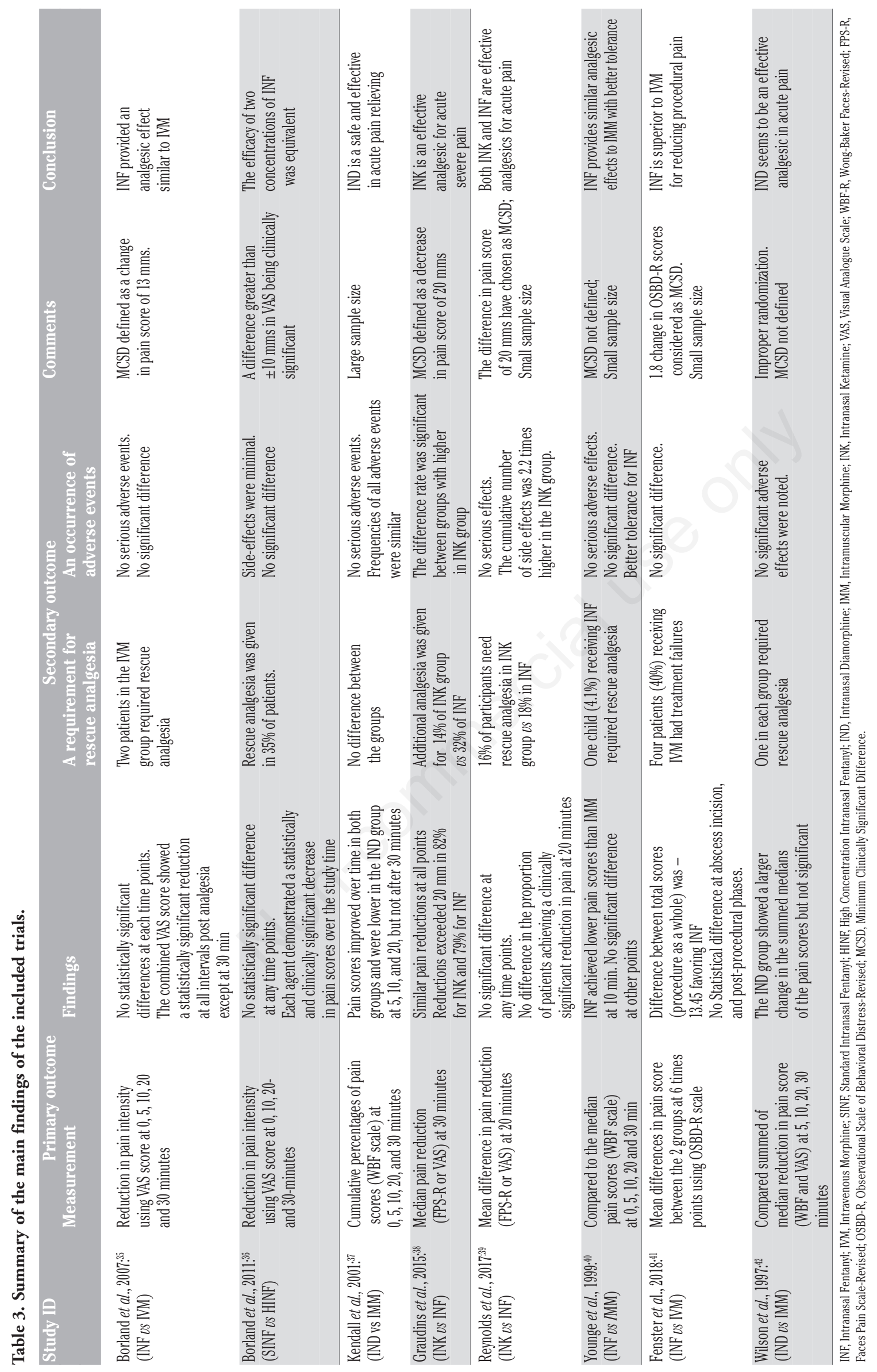


scores between the two treatment arms for each time point. However, when the VAS scores were combined (to form an overall score for each time point), he found statistically significant reductions at 5 minutes post analgesia of $20 \mathrm{~mm}(\mathrm{P}=0.000)$, at $10 \mathrm{~min}-$ utes of $4 \mathrm{~mm}(\mathrm{P}=0.012)$, and at 20 minutes of $8 \mathrm{~mm}(\mathrm{P}=0.000)$ but no further significant reductions beyond this time point.

Two trials studied IN diamorphine (IND) at a dose of 0.1 $\mathrm{mg} / \mathrm{kg}$ against IM morphine at a dose of $0.2 \mathrm{mg} / \mathrm{kg}$ and favored IND. Kendall ${ }^{37}$ reported that the onset of pain relief was faster in the diamorphine spray group than in the morphine group and found statistically significant lower pain scores in the spray group at 5 , 10 , and 20 minutes after treatment but not after 30 minutes. Similarly, Wilson ${ }^{42}$ reported that the IND group showed a broader change in the summed medians of the pain scores than the IM morphine group ( $9 v s 8)$ at 30 minutes, though this was not statistically significant.

When compared head to head, IN ketamine and fentanyl have been found to have similar pain reduction in children with moderate to severe pain from limb injury in 2 trials. Graudins et al. ${ }^{38}$ found similar pain reductions between groups at all points (i.e., 0 , 15,30 , and 60 minutes after intervention). At the primary endpoint of 30 minutes, he had found clinically significant reductions in VAS ratings with approximately $80 \%$ of subjects in both groups exceeding the defined MCSD of $20 \mathrm{~mm}$. Further, both treatments provided a considerable analgesic effect to 60 minutes post administration. Similarly, Reynolds et al. ${ }^{39}$ demonstrated no difference in efficacy between the two drugs either at $20 \mathrm{~min}$ or at other time points. However, thirty $(77 \%)$ subjects in the ketamine group and $35(80 \%)$ in the fentanyl group achieved a clinically significant reduction in pain at 20 minutes (risk difference $=-3 \%$ [95\% CI =$20 \%$ to $15 \%$ ]; $\mathrm{P}=0.77$ ) but no difference in the magnitude of change in pain score over time between the two treatment groups.

\section{A requirement for rescue analgesia}

All included trials reported the use of rescue analgesia in a proportion of 0 to $35.4 \%$ of patients who received IN analgesia. Data on the specific rescue agent and protocol used were reported in only five studies ${ }^{35-38,42}$ and were not specified in most studies regarding the used doses. The majority of studies offered rescue analgesia from $20 \mathrm{~min}$ onwards after the intervention. Only Reynolds's trial presented data on the amount of rescue analgesia consumed by the patients.

Borland $(2011)^{36}$ was the only one who described a statistically significant difference in the number of patients who required rescue analgesia. In this trail, rescue IV morphine was given to 67 of 189 patients (42 patients [41.1\%] received SINF, vs 25 [27.4\%] received HINF). The SINF group had significantly $(\mathrm{P}=0.028)$ more additional analgesia than HINF. Fenster found that the number of patients requiring rescue analgesia in the IN group was lower as compared to its comparator groups ( $0 v s 4)$. However, the sample size of this study was tiny. In contrast, Graudins and Reynolds concluded that patients who received IN Fentanyl required more rescue analgesia than those received IN ketamine but with no statistically significant difference. The rate of rescue analgesia was $32 \%$ in INF vs $14 \%$ in INK in Graudins's trial. Fifteen patients in Reynolds study required additional opioid rescue analgesia during the ED stay; seven patients in the ketamine group (16\%) and eight patients in the fentanyl group (18\%; risk difference=-2\% [95\% $\mathrm{CI}=-18 \%$ to $14 \%])$. No differences or data reported in the remaining studies.

\section{An occurrence of adverse events with intranasal drug delivery}

Based on patients or parental perceptions, tolerance of IN anal- gesic was found to be significantly better than tolerance of intramuscularly administered morphine in three trials..$^{37,40,42}$ All included trials reported a minimum or no side effects for IN analgesia. No one study reported any serious adverse events (e.g., opiate toxicity) or death.

The most common reported side effects were bad taste, drowsiness, nausea, vomiting, and itching nose. The frequencies of adverse events were similar between IN analgesia and alternative intervention groups. For example, $24 \%$ of patients who received IND spray in the Kendall trial had some adverse events compared to $19 \%$ of patients who received IM morphine. Although 84 serious adverse events were reported in this study, all were mild except for one in the spray group that was considered severe (abdominal pain and vomiting). However, two studies reported significantly higher incidences of adverse events when compared IN interventions to each other. All patients $(100 \%)$ of IN ketamine group vs $61 \%$ of the IN Fentanyl group in Reynolds trial and $78 \%$ vs $40 \%$ in Graudins's trial were found to have some adverse events.

\section{Discussion}

This literature review located and assessed eight published articles (7 RCTs and one QRC) that evaluated the safety and efficacy of IN analgesic for acute pain in children in PEDs.

\section{Summary of evidence}

Based on the findings of this review, three IN analgesics found to be safe, have equivalent or superior analgesic effects, and better tolerance than parenteral morphine in children who presented to PED with limb fracture or cutaneous abscess required incision and drainage. Besides, this review found that the associated adverse effects were infrequently reported and, when present, were minor and transient and did not need any intervention. No evidence of significant adverse events (e.g., opiate toxicity, anaphylaxis) or death was associated with the administration of any IN analgesia in any of the included studies.

The current evidence seems to show that IN fentanyl, diamorphine, and ketamine have an accepted efficacy for the treatment of moderate to severe traumatic and procedural pain in the PED. Similar results were also reported in a Cochrane review in 2014. ${ }^{28}$ Based on three trails, Murphy et al. ${ }^{28}$ concluded that INF might be an effective analgesic in painful conditions. Likewise, another systemic review by Poonai ${ }^{43}$ supports the result of this review regarding the effectiveness of IN ketamine. He concluded that IN ketamine administration is well tolerated and without serious adverse effects during procedural sedation and analgesia in children.

The majority of trials enrolled children aged three years or more with clinically deformed closed long bone fractures. No data in included studies investigate the use of any one of retrieved nasal analgesics in children less than three years.

It is important to note that all studies demonstrated equivalence in pain scores reduction at all intervals during the ED stay; however, significant pain relief was not last beyond $10 \mathrm{~min},{ }^{40} 20 \mathrm{~min},{ }^{35}$ and 30 minutes. ${ }^{36,37,42}$ Though, pain rating reduction was maintained to 60 minutes in two studies. ${ }^{38,39}$ Additionally, it is thought that slower absorption of intranasal medications in comparison to IV administration, would most likely make their efficacy slower than IV analgesics, especially 5 minutes post analgesia. However, the results of Borland (2007) $)^{35}$ and Fenster ${ }^{41}$ studies showed no statistically significant difference in median pain score between the two agents at any of the studies time points, including 5 minutes. Therefore, intranasal analgesia may decrease the time to pain relief 
and the time to analgesia administration.

As the studies comparing IN ketamine and fentanyl analgesia are sparse and limited, existence of 2 high-quality pieces of evidence in this review regarding head to head comparisons of IN ketamine $v s$ IN fentanyl, make us confident to conclude that there is a no significant difference in pain reduction when the various forms of IN analgesia would compare for control of acute pain in PED. However, adverse effects seem to be more frequent with ketamine.

As mentioned above, the frequencies of adverse events were limited, and when they occurred were all relatively mild. Based on this finding, we could conclude that the IN analgesia has an acceptable safety profile. However, the majority of included studies have small sample sizes, make this conclusion unconfident. Therefore, a large, multicenter study in children would be required to determine the exact rate of their side effects. Besides, safety studies have not been conducted to look at long-term effects on the nasal mucosa.

Due to incomplete data regarding the specific rescue agent used were not specified in most studies, various time points at which rescue was offered, and a lack of enough data on the amount of rescue analgesia consumed by patients, it was unable to evaluate the potential impact rescue analgesia had on the reported outcomes of this review. Even though the majority of studies offered rescue analgesia either at $20 \mathrm{~min}$ or $30 \mathrm{~min}$ after administration of the intervention drug, this finding suggested that physicians should expect that patients who receive IN analgesia may require to receive rescue analgesia after 30 minutes due to the short duration of action. Further, as two trials ${ }^{35,41}$ reported that the percentage of patients requiring rescue analgesia in the INF groups was significantly lower than their comparator groups, it should be noted that those trial used either higher dose $(2 \mathrm{ug} / \mathrm{kg})$ or concentration (300 $\mathrm{mg} / \mathrm{mL}$ ) of fentanyl.

Admittedly, among presented studies, it does not exist a standard dosage per $\mathrm{Kg}$ (minimum effective dosage of which would be the suggested one to obtain the best result with less adverse events) in children. Furthermore, also at higher dosages IN therapy remains safe with lower side effects.

\section{Quality of included evidence}

The overall quality of the included evidence ranged from very low to high. Some limitations were noted in the design and implementation of all included studies. Despite Kendall's trial ${ }^{37}$ downgraded by $(-1)$ as has a high risk of detection and performance bias, the significance levels for tests of difference in outcomes between groups were upgraded to high quality. Younge's study ${ }^{40}$ was limited in design by the open nature of the trial and did not meet all the criteria for a good quality study, suggesting a likely potential source of bias. The comparative efficacy of the two drugs in Reynolds' study ${ }^{39}$ was an exploratory measure as the study was not powered to detect a difference in this outcome. Borland (2007) ${ }^{35}$ used a convenience sample for enrolments that were dependent on the identification of suitable participants at triage. No record was kept of potential participants who were not enrolled so that no conclusion can be drawn about potential selection bias. Enrolment in Borland (2011) ${ }^{36}$ was not compulsory but was actively encouraged by study investigators. Not all patients were able to be screened for inclusion in the study, and this might affect external validity. However, based on reported similarities between cohorts of included and non-included patients, this potential source of selection bias was minimized. Despite prior specific criteria for inclusion, there was potential selection bias based on the need for opiate analgesia. Fenster's study has a high risk of detection bias, as the treating physician was not blinded to the study drug, and it has significant imprecision due to few participants were enrolled in each arm.
Finally, Wilson describes a non-random component in the sequence generation process, the patients were randomized according to their hospital number, without blinding of participants or outcome assessors, made it at very high risk for selection, performance, and detection bias. Neither indirectness of evidence nor unexplained heterogeneity or inconsistency of results were identified in any included study. The overall risk of publication bias was thought to be low in all included studies.

\section{Limitations}

This literature review has certain limitations. Only trials that were written in the English language were included. To obtain the highest possible internal trial validity, only double-blinded RCTs or quasi-RCT were chosen, this could potentially result in a high trial exclusion rate and might be a limitation of this review. However, in the inclusion of trials with no adequate blinding, the risks of false-negative and false positive results are difficult to assess and could potentially result in an inaccurate conclusion. Further, the majority of the included studies were either openlabel, single-blinded, had a small sample size, or conducted in a single center. Another limitation was in the quality of the studies and the use of a single reviewer to grade them. Five of the studies were high quality while remaining studies were either moderate, ${ }^{40}$ low, ${ }^{41}$ or very low-quality ${ }^{42}$ evidence. Furthermore, the authors were not contacted regarding some missed trial information. Due to significant heterogeneity in the methodology and outcomes assessment of the included studies, hence only a narrative synthesis of the results was reported.

Studies evaluate pain scores applied up to 30 minutes from drug administration and not later. In this way, the antalgic effect of the drug can only be evaluated in the initial acute phase of the pain but not in the following one: comparison, for example, with morphine, is applicable only for initial 30 minutes when fentanyl has the fastest action but for the prolonged half-life of morphine its IV or IM action after 30 minutes is probably higher than IN drugs. Therefore, also, evaluation time has to be considered as a limitation of the study to the real evaluation of the antalgic effects of drugs.

I believe that the findings of this review are valid and widely applicable. However, all included trials were conducted in developed countries. Therefore, the findings may not be generalizable to all PED pediatric patients in all countries.

\section{Conclusions}

This review identified eight articles that discussed the IN analgesia as a possible route of analgesia in the PED. While no paper was entirely perfect, the findings support that IN analgesia may be an effective analgesic for the treatment of the children (3-18 years) with acute moderate to severe pain, and its administration appears to cause minimal adverse effects.

\section{References}

1. Johnston C, Gagnon P, Fullerton M, et al. One-week survey of pain intensity on admission to and discharge from the emergency department: a pilot study. J Emerg Med 1998;16:377-82.

2. Friedland L, Pancioli A, Duncan K. Pediatric emergency department analgesic practice. Pediatr Emerg Care 1997;13:103-6. 
3. Murphy A, McCoy S, O'Reilly K, et al. A prevalence and management study of acute pain in children attending emergency departments by ambulance. Prehosp Emerg Care 2016;20:528.

4. McGrath P, Walco G, Turk D, et al. Core outcome domains and measures for pediatric acute and chronic/recurrent pain clinical trials: PedIMMPACT recommendations. J Pain 2008;9:77183 .

5. Hennes H, Kim M, Pirrallo R. Prehospital pain management: a comparison of providers' perceptions and practices. Prehosp Emerg Care 2005;9:32-9.

6. Pate J, Blount R, Cohen L, Smith A. Childhood medical experience and temperament as predictors of adult functioning in medical situations. Child Health Care 1996;25:281-98.

7. Anand KJ. Pain, plasticity, and premature birth: A prescription for permanent suffering? Nat Med 2000;6:971-3.

8. Kennedy R, Luhmann J, Zempsky W. Clinical implications of unmanaged needle-insertion pain and distress in children. Pediatrics 2008;122:S130-3.

9. Kassam-Adams N. Introduction to the special issue: posttraumatic stress related to pediatric illness and injury. J Pediatr Psychol 2006;31:337-42.

10. Rupp T, Delaney K. Inadequate analgesia in emergency medicine. Ann Emerg Med 2004;43:494-503.

11. Schug S, Palmer G, Scott D, et al. Working Group of the Australian and New Zealand College of Anesthetists and Faculty of Pain Medicine. The pediatric patient. In: Schug SA, Palmer GM, Scott DA, et al., eds. Acute pain management: scientific evidence. 4th ed. Melbourne: ANZCA \& FPM. pp 409514.

12. Fein J, Zempsky W, Cravero J. Relief of pain and anxiety in pediatric patients in emergency medical systems. Pediatrics 2012;130:e1391-405.

13. Baker DW. Joint Commission Statement on Pain Management: The Joint Commission. Oakbrook Terrrace, IL: The Joint Commission; 2012. Available from: https://www.jointcommission.org/joint_commission_statement_on_pain_management/ Accessed: September 28, 2018.

14. Selbst S, Henretig F. The treatment of pain in the emergency department. Pediatr Clin North Am 1989;36:965-78.

15. Probst B, Lyons E, Leonard D. Factors affecting emergency department assessment and management of pain in children. Pediatr Emerg Care 2005;21:298-305.

16. Elder K, Rice S, Dean C, Piper C. Addressing the differences by race in analgesia use among pediatric patients attending emergency departments. J Pediatr 2014;165:434-6.

17. Czarnecki M, Simon K, Thompson J, et al. Barriers to pediatric pain management: a nursing perspective. Pain Manag Nurs 2011;12:154-62.

18. Alexander J, Manno M. Underuse of analgesia in very young pediatric patients with isolated painful injuries. Ann Emerg Med 2003;41:617-22.

19. WHO. WHO Normative Guidelines on Pain Management. Report of a Delphi study to determine the need for guidelines and to identify the number and topics of guidelines that should be developed by WHO. Report prepared by Prof Neeta Kumar. Geneva, Switzerland: World Health Organization; 2007.

20. Pancekauskaitè G, Jankauskaitè L. Paediatric pain medicine: pain differences, recognition, and coping acute procedural pain in the pediatric emergency room. Medicina (Kaunas) 2018;54:94.

21. Oakley E, Barnett P, Babl FE. Backslap versus no back slab for immobilization of undisplaced supracondylar fractures: a ran- domized trial. Pediatr Emerg Care 2009;25:452-6.

22. Mills E, Craig S, Oakley E. Busted! Management of pediatric upper limb fractures: not all that it's cracked up to be. Emerg Med Australas 2014;26:384-91.

23. College of Emergency Medicine Clinical Effectiveness Committee. Management of pain in children (Rev July 2013). London: The Royal College of Emergency Medicine; 2013. Available from: https://www.rcem.ac.uk/docs/RCEM\% 20 Guidance/RCEM $\% 20$ Pain $\% 20$ in $\% 20$ Children $\% 20$ \%20Best\%20Practice\%20Guidance\%20(REV\%20Jul\%20201 7).pdf Accessed: September 29, 2018.

24. Krauss B, Calligaris L, Green S, Barbi E. Current concepts in the management of pain in children in the emergency department. Lancet 2016;387:83-92.

25. Gizurarson S. Anatomical and histological factors affecting intranasal drug and vaccine delivery. Curr Drug Delivery 2012;9:566-82.

26. Cauna N, Hinderer K. Fine structure of blood vessels of the human respiratory mucosa. Ann Otol Rhinol Laryngol 1969;78:865.

27. Williams P, Warwick R, eds. Gray's anatomy. 36th ed. Edinburgh: Churchill Livingstone; 1980.

28. Murphy A, O’Sullivan R, Wakai A, et al. Intranasal fentanyl for the management of acute pain in children. Cochrane Database Syst Rev 2014;10:CD009942.

29. Walbergh E, Wills R, Eckliert J. Plasma concentrations of midazolam in children following intranasal administration. Anesthesiology 1991;74:233.

30. Sarkar MA. Drug metabolism in the nasal mucosa. Pharm Res 1992;9:1-9.

31. Marttin E, Nicolaas G, Schipper J, et al. Nasal mucociliary clearance as a factor in nasal drug delivery. Adv Drug Del Rev 1997;29:13-38.

32. Moher D, Liberati A, Tetzlaff J, et al. Preferred reporting items for systematic reviews and meta-analyses: The PRISMA statement. Ann Intern Med 2009;151:264-9.

33. Guyatt G, Oxman A, Vist G, et al. GRADE: an emerging consensus on rating quality of evidence and strength of recommendations (Grading of Recommendations Assessment, Development and Evaluation). BMJ 2008;26:924-6.

34. Higgins J, Green S, eds. Cochrane handbook for systematic reviews of interventions version 5.1 [updated March 2011]. The Cochrane Collaboration; 2011. Available from: http://handbook.cochrane.org

35. Borland M, Jacobs I, King B, O'Brien D. A randomized controlled trial comparing intranasal fentanyl to intravenous morphine for managing acute pain in children in the emergency department. Ann Emerg Med 2007;49:335-40.

36. Borland M, Milsom S, Esson A. Equivalency of two concentrations of fentanyl administered by the intranasal route for acute analgesia in children in a paediatric emergency department: a randomized controlled trial. Emerg Med Australas 2011;23:202-8.

37. Kendall J, Barnaby R, Victoria S. A multicenter randomized controlled trial of nasal diamorphine for analgesia in children and teenagers with clinical fractures. BMJ 2001;322:26.

38. Graudins A, Meek R, Egerton-Warburton D, et al. The PICHFORK (Pain in Children Fentanyl or Ketamine) trial: a randomized controlled trial comparing intranasal ketamine and fentanyl for the relief of moderate to severe pain in children with limb injuries. Ann Emerg Med 2015;65:248-54.

39. Reynolds S, Bryant K, Studnek J, et al. Randomized controlled feasibility trial of intranasal ketamine compared to intranasal 
fentanyl for analgesia in children with suspected extremity fractures. Acad Emerg Med 2017;24:1430-40.

40. Younge $\mathrm{P}, \mathrm{Nicol} \mathrm{M}, \mathrm{Kendall} \mathrm{J}$, et al. A prospective randomized pilot comparison of intranasal fentanyl and intramuscular morphine for analgesia in children presenting to the emergency department with clinical fractures. Emerg Med 1999;11:90-4.

41. Fenster D, Dayan P, Babineau J, et al. Randomized trial of intranasal fentanyl versus intravenous morphine for abscess incision and drainage. Pediatr Emerg Care 2018;34:607-12.

42. Wilson J, Kendall J, Cornelius P. Intranasal diamorphine for paediatric analgesia: assessment of safety and efficacy. J Accid Emerg Med 1997;14:70-2.

43. Poonai N, Canton K, Ali S, et al. Intranasal ketamine for procedural sedation and analgesia in children: A systematic review. PLoS One 2017;12:e0173253. 